\title{
Energy loss of helium ions in zinc
}

\author{
G. H. Lantschner, ${ }^{1}$ J. C. Eckardt, ${ }^{1}$ A. F. Lifschitz, ${ }^{1}$ N. R. Arista, ${ }^{1}$ L. L. Araujo, ${ }^{2}$ P. F. Duarte, ${ }^{2}$ J. H. R. dos Santos, ${ }^{2}$ \\ M. Behar, ${ }^{2}$ J. F. Dias, ${ }^{2}$ P. L. Grande, ${ }^{2}$ C. C. Montanari, ${ }^{3}$ and J. E. Miraglia ${ }^{3}$ \\ ${ }^{1}$ Centro Atómico Bariloche, Comisión Nacional de Energía Atómica, 8400 S. C. de Bariloche, Argentina \\ ${ }^{2}$ Instituto de Física da Universidade Federal do Rio Grande do Sul, Avenida Bento Goncalves 9500, 91501-970, \\ Porto Alegre, RS, Brazil \\ ${ }^{3}$ Instituto de Astronomía y Física del Espacio, Casilla Correo 67, Sucursal 28, 1428 Buenos Aires, Argentina
}

(Received 16 December 2003; published 9 June 2004)

\begin{abstract}
The energy loss of helium ions in zinc has been measured in the energy range from 37.5 to $1750 \mathrm{keV} / \mathrm{amu}$ using the transmission technique and the Rutherford backscattering method. In addition, calculations using the extended Friedel sum rule, the unitary convolution approximation, and the local plasma approximation have been performed. The contributions of the inner-shell and valence electrons to the total energy loss are separately evaluated. The measurements and calculations are in good agreement over an extended range of energies, and both of them yield stopping values higher than those provided by SRIM 2003.
\end{abstract}

DOI: 10.1103/PhysRevA.69.062903

PACS number(s): $34.50 . \mathrm{Bw}$

\section{INTRODUCTION}

Measurements of the stopping power of light ions in zinc are very scarce despite the widespread technological use of this metal and the relevance of the stopping power for the evaluation of radiation damage, the determination of depth profiles in ion implantation, and other applications. Some of the stopping power compilations [1,2] do not include $\mathrm{Zn}$ data at all. The well-known tabulation of Ref. [3] only contains data for protons, whereas for helium ions only estimated data are included [4]. The present version of this tabulation [5] only includes experimental data up to $500 \mathrm{keV} / \mathrm{amu}$ (see also Ref. [6]). This scarcity of data is mainly due to difficulties in the preparation of thin zinc samples by evaporation given its condensation properties.

Here we present measurements of helium ions in zinc over an energy range from 37.5 to $1750 \mathrm{keV} / \mathrm{amu}$ together with calculations covering a wide range of energies, including the interesting region around the stopping power maximum. The measurements were made by two different techniques: the transmission method between 37.5 and $150 \mathrm{keV} / \mathrm{amu}$, and the Rutherford backscattering (RBS) technique between 92.5 and $1750 \mathrm{keV} / \mathrm{amu}$. The target thicknesses were determined by energy loss measurements of protons at $200-1000 \mathrm{keV}$ and using the stopping power tables of Ref. [3], which is equivalent to normalize the present measurements to these tabulated values. Together with previous measurements made in one of the laboratories (Centro Atómico Bariloche) [7] the present results provide a fairly complete set of data covering a wide energy range extending from 0.75 to $1750 \mathrm{keV} / \mathrm{amu}$.

In the calculations we present here, the contributions to the stopping of the valence electrons and the target inner shells electrons are evaluated separately. For the valence electrons we use the generalized Friedel sum rule formalism, while the core electrons contribution is calculated by means of the unitary convolution approximation and the local plasma approximation.

\section{EXPERIMENTAL PROCEDURE}

The experiments at lower and intermediate energies (37.5 to $150 \mathrm{keV} / \mathrm{amu}$ ) were carried out at the Centro Atómico Bariloche $(\mathrm{CAB})$ employing the transmission method. At higher energies, between 92.5 and $1750 \mathrm{keV} / \mathrm{amu}$, the experiments were performed at the Instituto de Física of the Universidade Federal do Rio Grande do Sul (IF-UFRGS) using the Rutherford backscattering (RBS) technique.

\section{A. Transmission experiments}

The self-supported foils used in the transmission measurements were made by evaporation under clean vacuum conditions on a very smooth plastic substrate [8] after previous deposition of a very small amount of gold to allow the condensation of Zn. Subsequently, the plastic substrate was dissolved so as to make eventual residues undetectable in energy loss experiments. The mean foil thicknesses $\langle x\rangle$ were $22 \pm 1 \mathrm{~nm}$ and have been determined by energy loss measurements of protons at $200 \mathrm{keV}$ and the use of the stopping power values from Ref. [3]. To characterize the foil inhomogeneity we employed an ion beam analysis using $\mathrm{H}^{+}$and $\mathrm{He}^{+}$ beams [9], which allows the determination of an upper bound for the standard deviation $\sigma$ of the foil thickness distribution. The resulting upper bounds for the roughness coefficients $\rho=\sigma /\langle x\rangle$ ranged from $10 \%$ to $14 \%$.

In order to extend the energy range of the transmission measurements we used single and double charged ions of the ${ }^{3} \mathrm{He}$ isotope. The beams were generated by electrostatic acceleration of ${ }^{3} \mathrm{He}^{+}$and ${ }^{3} \mathrm{He}^{2+}$ ions produced in a rf ion source. Electrostatic focusing, magnetic mass selection stages, and collimation defined the final beam. The foils were mounted on a movable holder which allowed changing the targets and removing them from the beam path during the operation of the accelerator. The energy analysis was performed by an electrostatic analyzer with $0.3 \%$ full width at half maximum resolution, positioned in the forward beam direction. The particles were detected by a discrete dynode 


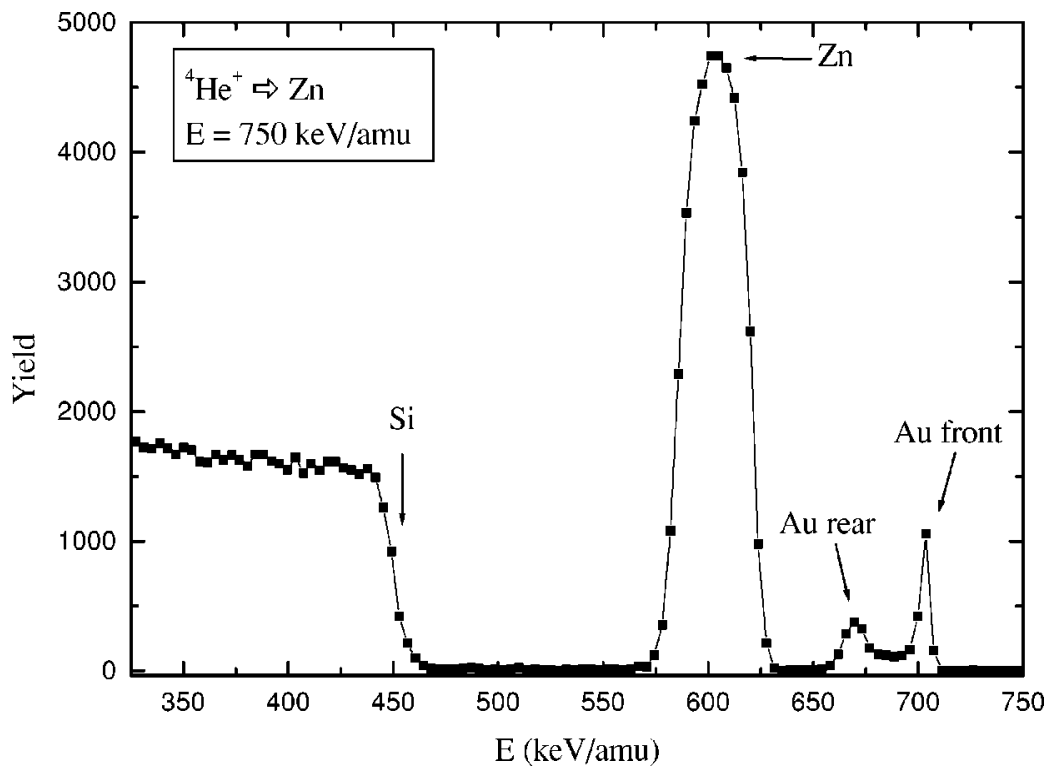

FIG. 1. RBS spectrum of the $150 \mathrm{~nm}$ thick $\mathrm{Zn}$ sample obtained with a $750 \mathrm{keV} / \mathrm{amu} \mathrm{He}^{+}$beam.

electron multiplier followed by conventional pulse counting electronics. Spectra were recorded by a multichannel scaler with channels switched synchronously with the energy analyzer plate potential.

Foil thickening of the self-supporting foils by beam bombardment [10] was held within negligible limits by using a low ion current density of $\sim 10^{-9} \mathrm{~A} / \mathrm{cm}^{2}$, and irradiation times of less than 2 min per spectrum. In this way no change in foil characteristics could be detected during the time of measurements.

At the energies of this experiment and with the employed foil thicknesses, the transmission experiment spectra were nearly Gaussian, so that the mean energy was determined directly from them.

\section{B. Rutherford backscattering experiments}

In order to perform the He energy loss measurements using this technique, we have used a set of three multilayer $\mathrm{Au}(5 \mathrm{~nm}) / \mathrm{Zn} / \mathrm{Au}(5 \mathrm{~nm})$ films which were vapor-deposited on Si wafers. The inner Au film was necessary since $\mathrm{Zn}$ does not have good adherence on Si. The outer Au film was deposited in order to be used as an energy marker (together with the inner one) during the RBS measurements. In this way we have two independent ways to determine the energy loss from the measured spectra.

The thickness determination of the $\mathrm{Zn}$ films was performed through proton energy loss measurements, using again the stopping power values for hydrogen in $\mathrm{Zn}$ from the Andersen and Ziegler tables [3]. With this aim we have used beams of different energies (from 400 to $1000 \mathrm{keV}$ ) and different geometries. The backscattered particles were detected with a Si surface barrier detector placed at $120^{\circ}$ with respect to the beam direction. The resolution of the detector plus the electronic system was better than $7 \mathrm{keV}$. Following this procedure we have determined that the thicknesses of the $\mathrm{Zn}$ films were $46 \pm 2,75 \pm 3$, and $150 \pm 7 \mathrm{~nm}$, respectively. The main source of the quoted errors arises from the uncertainty in the $\mathrm{H}$ stopping powers.
The determination of the stopping powers of $\mathrm{He}$ in $\mathrm{Zn}$ was done by measuring energy losses and using the previously determined film thickness. In the present case, the detector plus electronic resolution was better than $13 \mathrm{keV}$. The energy range covered by the present measurements reached from 92.5 up to $1750 \mathrm{keV} / \mathrm{amu}$. Each one of the $\mathrm{Zn}$ films was used for a given He energy range. A typical RBS spectrum is shown in Fig. 1 for a $750 \mathrm{keV} / \mathrm{amu} \mathrm{He}^{+}$beam. At this energy it is possible to see the two peaks corresponding to the $\mathrm{Au}$ films that enclose the $\mathrm{Zn}$ film.

\section{RBS data analysis}

Given the expression

$$
[\epsilon]=\Delta E / x
$$

with $[\epsilon]$ being the energy-loss factor [11] of He in $\mathrm{Zn}$ and $\Delta E$ the measured energy loss corresponding to the width $x$ of the $\mathrm{Zn}$ film, it is possible to determine the stopping power of $\mathrm{He}$ in $\mathrm{Zn}$ through the relation between the energy-loss factor and the energy loss per unit length $d E / d x$ (in the surface energy approximation [11]):

$$
[\epsilon(\mathrm{He})]=\left.\frac{K}{\cos \theta_{1}} \frac{d E}{d x}\right|_{E_{0}}+\left.\frac{1}{\cos \theta_{2}} \frac{d E}{d x}\right|_{K E_{0}},
$$

where $K$ is the kinematic factor and $\theta_{1}$ and $\theta_{2}$ are the angles between the sample's normal with the incoming beam and the detector's position, respectively. As can be observed from expressions (1) and (2), at least two measurements of $\Delta E$ should be done (performed at different geometries) in order to determine the energy losses $d E / d x$ at the energies $E_{0}$ and $K E_{0}$, respectively.

Expression (2) can be rewritten in the following way:

$$
\left.\frac{d E}{d x}\right|_{K E_{0}}=\left.m \frac{d E}{d x}\right|_{E_{0}}+n
$$

with $m$ being a function of $\cos \theta_{1}, \cos \theta_{2}$, and the kinematic factor $K$, and $n$ being a function of $\cos \theta_{2}$ and the correspond- 


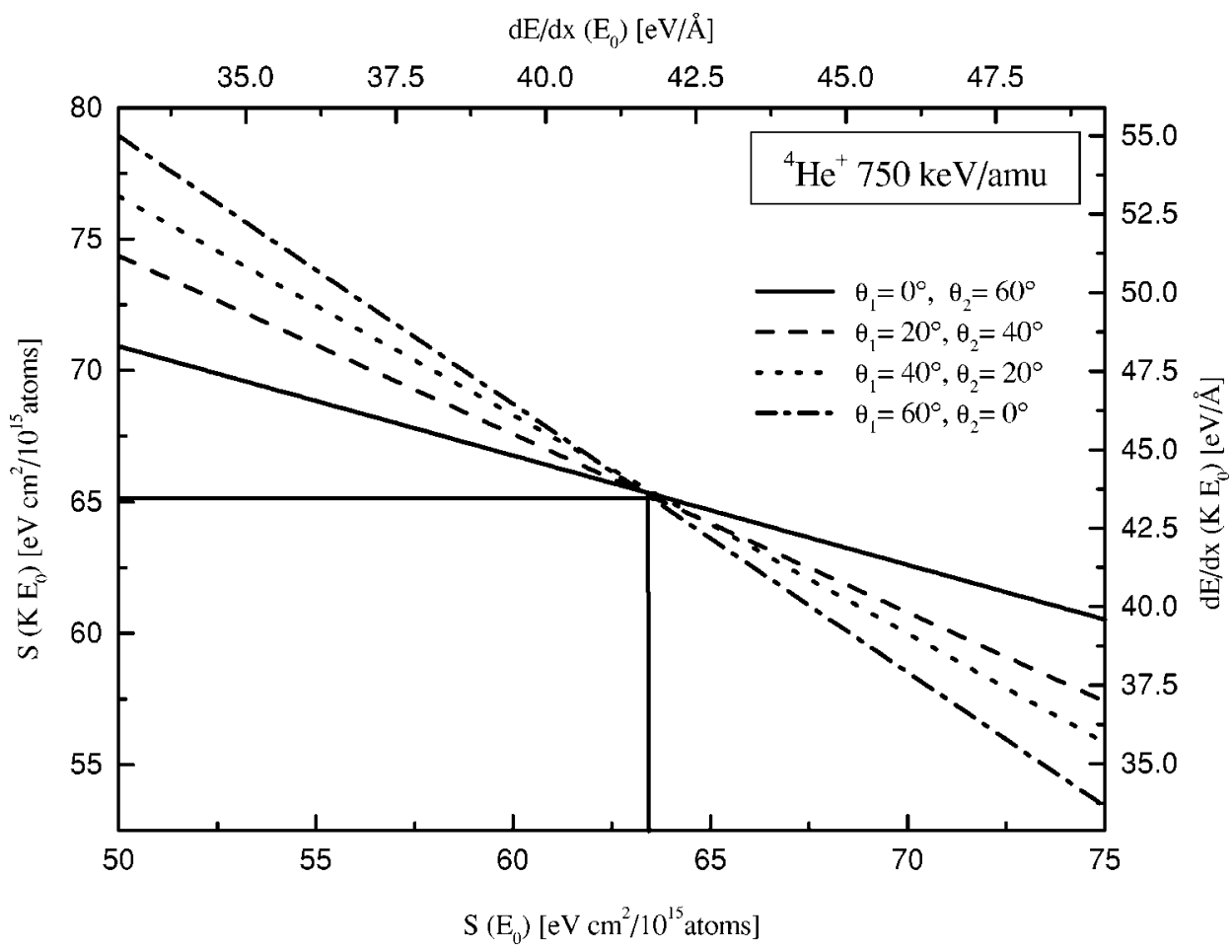

FIG. 2. Intersection of the straight lines [Eq. (3)] for the $750 \mathrm{keV} / \mathrm{amu}$ stopping power measurements at different geometries. The bold lines indicate the energy loss values obtained for $E_{0}$ (horizontal axis) and $K E_{0}$ (vertical axis).

ing energy-loss factor $S$. So each measurement of $S$ defines a straight line in the $d E /\left.d x\right|_{E_{0}}$ and $d E /\left.d x\right|_{K E_{0}}$ plane. By changing both angles, a family of straight lines can be obtained. The intersection of the straight lines gives the corresponding stopping powers at $E_{0}$ and $K E_{0}$. We have performed four measurements under different geometrical conditions for each energy $E_{0}$. A typical plot for $750 \mathrm{keV} /$ amu helium ions is displayed in Fig. 2.

It should be noted that for each energy there are at most six sets of different $d E /\left.d x\right|_{E_{0}}$ and $d E /\left.d x\right|_{K E_{0}}$ values which arise from the several possible intersections between the four straight lines. Then we have taken the corresponding mean values and those are the ones further quoted.

The errors of the present measurements were estimated taking into account the statistical dispersion and the reported uncertainties in the stopping power for protons of Ref. [3], which in this case are 5\%. For protons in $\mathrm{Zn}$ the values from the referred tables stem from interpolation of the curves corresponding to neighboring target elements, however, at the energies used for our normalization, they show a close agreement with previous experimental data also plotted in [3].

\section{THEORETICAL CALCULATIONS}

The valence electron and inner-shell electron contributions to the energy loss are evaluated separately using higherorder (nonperturbative) methods. The first contribution is calculated in the binary collisions framework using the extended Friedel sum rule-transport cross section (EFSR-TCS) scheme $[12,13]$. In this approach, the valence electrons are considered as a homogeneous electron gas with Wigner-Seitz radius $r_{s}$. The scattering potential seen by target electrons around the moving ion is modeled as a sum of a core potential (generated by the electrons bound to the projectile) and a screening potential (generated by the target valence electrons), that is

$$
\phi_{\text {ion }}(r)=q \phi_{\text {core }}(r)+\left(Z_{1}-q\right) \phi_{s}(r)
$$

where $q$ is the projectile charge state and $Z_{1}$ the atomic number $\left(Z_{1}=2\right.$ for $\left.\mathrm{He}\right)$. The core potential model used here is an adaptation of the atomic Molière potential to the case of ions [14]. For the screening part we use a simple Yukawa potential, $\phi_{s}(r)=\exp (-\alpha r) / r$, where the value of $\alpha$ is adjusted for each energy using the extended Friedel sum rule [12]. The results of this method are not very sensitive to the assumed model for the ion potential provided that a self-consistent adjustment of the parameters is made using the EFSR, as it was shown previously [12-14]. We assume that the charge state of the ion is close to the charge state measured after emerging from the solid [15]. The values of $q$ are given by the empirical fitting of Schiwietz and Grande [16], which agree closely with the experimental data of emerging charge [17]. For $r_{s}$ we use the value inferred from plasma frequency measurements, that is $r_{s}=2$ [18].

The contribution to the energy loss of $\mathrm{Zn}$ inner shells is calculated using the unitary convolution approximation (UCA) method [19] from the program CASP [20]. The same charge-state values $q$ have been used as for the valence electron calculations. For the target parameters, the electronic density for each subshell was taken into account. For each sub shell, however, only a single oscillator strength and a mean transition energy was considered. The mean transfer energy was assumed to be equal to $\nu I_{s}$, with $I_{s}$ being the binding energy of the shell $s$ under consideration and 


$$
\nu=I_{\text {Bethe }} / \exp \left(\frac{1}{N} \sum_{s} n_{s} \ln \left(I_{s}\right)\right)
$$

where $n_{s}$ is the number of electrons of the shell $s$ and $N$ is the total number of electrons. For $\mathrm{Zn}$, the Hartree-Fock-Slater [20] values of $I_{s}$ were used $\left(I_{1 s}=9505 \mathrm{eV}, I_{2 s}=1160 \mathrm{eV}\right.$, $I_{2 p}=1029 \mathrm{eV}, I_{3 s}=135 \mathrm{eV}, I_{3 p}=92 \mathrm{eV}, I_{3 d}=18.3 \mathrm{eV}$, and $I_{4 s}=9.3 \mathrm{eV}$ ). This choice is consistent with the $I_{\text {Bethe }}$ from the Bethe formula. Here, we have used the ICRU value $I_{\text {Bethe }}$ $=330 \mathrm{eV}$ for solid $\mathrm{Zn}$. The UCA provides reliable energy loss values for bare and screened projectiles at high and intermediate energies. At lower energies the UCA results should overestimate the energy loss from the inner shells but, at these energies, the stopping is dominated by the valenceelectron contribution, which is evaluated through the EFSRTCS method.

We have also calculated the contribution of $\mathrm{Zn}$ inner-shell electrons by employing the dielectric formalism through the local plasma approximation (LPA)[21-23]. The dielectric formalism, usually employed to deal with collisions involving solid targets, allows us to take into account the electronic response in a dynamical way. The LPA considers that under certain conditions (perturbative limit, i.e., impact velocity higher than the velocity of the electrons in each shell), bound electrons react to the ion perturbation as free electrons in a screened potential. In this way, solid inner-shell electrons are described as a free electron gas of inhomogeneous density that is polarized and excited by the passing ion.

The spatial-dependent densities $n_{s}(r)$ of each shell of $\mathrm{Zn}$ (i.e., $1 s, 2 s, 2 p, 3 s, 3 p$, and $3 d$ ) are obtained from the atomic Hartree-Fock wave functions [24], following previous works in which the LPA showed very good agreement with experimental data $[22,23,25,26]$. We calculate the contribution of each shell of target electrons separately, and the total innershell energy loss as the addition of these contributions (shell to shell).

The stopping power of dressed projectiles (He ions in this case) is calculated in the LPA by replacing the protonelectron Coulomb potential by a screened one [27,28]. The Fourier transform of this potential has the same explicit form as the Coulomb potential with screened ion charge $F_{1}^{s c r}(k)$ $=Z_{1}-Z_{e}(k)$, which depends on the momentum transfer $k$. The term $Z_{e}(k)=\sum_{n=1}^{N}\left\langle\varphi_{n}\left|e^{i \mathbf{k} \cdot \mathbf{r}}\right| \varphi_{n}\right\rangle$ represents the screening of $Z_{1}$ by the $N$ bound electrons. It is calculated from the form factors of the electrons in the shells as if remaining frozen (electron excitations or loss are not taken into account). For large $k, F_{1}^{s c r}(k) \rightarrow Z_{1}$, while for $k \rightarrow 0, F_{1}^{s c r}(k) \rightarrow 0$. For the inner shells (i.e., $K$ and $L$ shells of $\mathrm{Zn}$ ), the main contribution comes from large $k$ so the screening of the ion by its passive electrons is not very relevant, since for close collisions at small impact parameters the target electrons interact with the projectile nucleus almost as a bare ion. On the other hand, for the outer shells ( $M$ shell of $\mathrm{Zn}$ ), the main contribution is in the region of small $k$ and the ion charge is strongly screened.

The contribution of each $n l$ shell of target electrons to the stopping power in the LPA is then expressed as

$$
S_{n l}=\frac{2}{\pi v^{2} n_{a t}} \int_{0}^{\infty} \frac{d k}{k} F_{1}^{s c r}(k) \int_{0}^{k v} \omega \operatorname{Im}\left[\frac{-1}{\varepsilon_{L P A}^{n l}(k, \omega)}\right] d \omega,
$$

where $\varepsilon_{L P A}^{n l}(k, \omega)$ is the dielectric function of the $n l$-shell electrons in the LPA, as given in Eq. (1) of Ref. [22].

The contribution of $\mathrm{Zn}$ inner shells to the stopping power of $\mathrm{He}^{2+}, \mathrm{He}^{+}$, and $\mathrm{He}^{0}$ in the LPA is calculated separately. The charge-state values of Schiwietz and Grande [16] are employed at each impact energy to get the total stopping power of He ions. In addition, we have calculated the "antiscreening" contribution to the stopping power which is due to the processes of excitation and ionization of the projectile electrons, folowing the approach of Ref. [23]. We have found that this contribution is very small (less than $1 \%$ ) and so this term was not included in the present comparisons.

\section{RESULTS}

The complete set of results measured at $\mathrm{CAB}$ and IFUFRGS is shown in Fig. 3. As it may be observed, in the region of overlap there is a good agreement between the transmission measurements and the RBS results. In the same figure, the results from the present calculations are depicted, showing the separate contributions of valence electrons and the total value, including the valence electrons from EFSRTCS and shell electrons from UCA and LPA models. One can observe a good agreement with the present experimental values in the whole energy range. As expected, the valence electrons dominate the stopping process at low energies, whereas at higher energies the main contribution to the energy loss comes from the inner-shell electrons. For very low energies, the stopping is mainly due to collisions with valence electrons. Indeed, we can see that the calculated values for the valence contribution approach previous experimental values [7] for $E \leqslant 2.5 \mathrm{keV} / \mathrm{amu}$.

It may be noted the relevance of using a nonlinear method (EFSR-TCS) [12,13] to describe the energy loss due to the valence electrons. This makes a considerable difference in the energy loss results at low energies. In Fig. 3 we also display the perturbative calculations following the dielectric formalism. As may be observed, at $10 \mathrm{keV} / \mathrm{amu}$ the nonlinear calculations yield values about four times larger than the perturbative ones, and approaches them for energies above $70 \mathrm{keV} / \mathrm{amu}$.

The comparison with the experimental stopping cross sections is possible by adding the contributions due to valence and inner-shell electrons. We present these total values following the two models mentioned in the previous section: the UCA calculations and the LPA results from the dielectric formalism for solids. Both curves display very good agreement with the experimental data.

The UCA results are displayed for energies above $17.5 \mathrm{keV} / \mathrm{amu}$, which should be the lower limit of validity of the model for He ions. Near the maximum of the stopping power, the UCA curve shows a better accord with the experimental data than the LPA curve. At low energies, the combination of LPA results for inner shells with the nonperturbative ones for the valence electrons give a very good accord with experimental data. Actually, the main contribution to the 


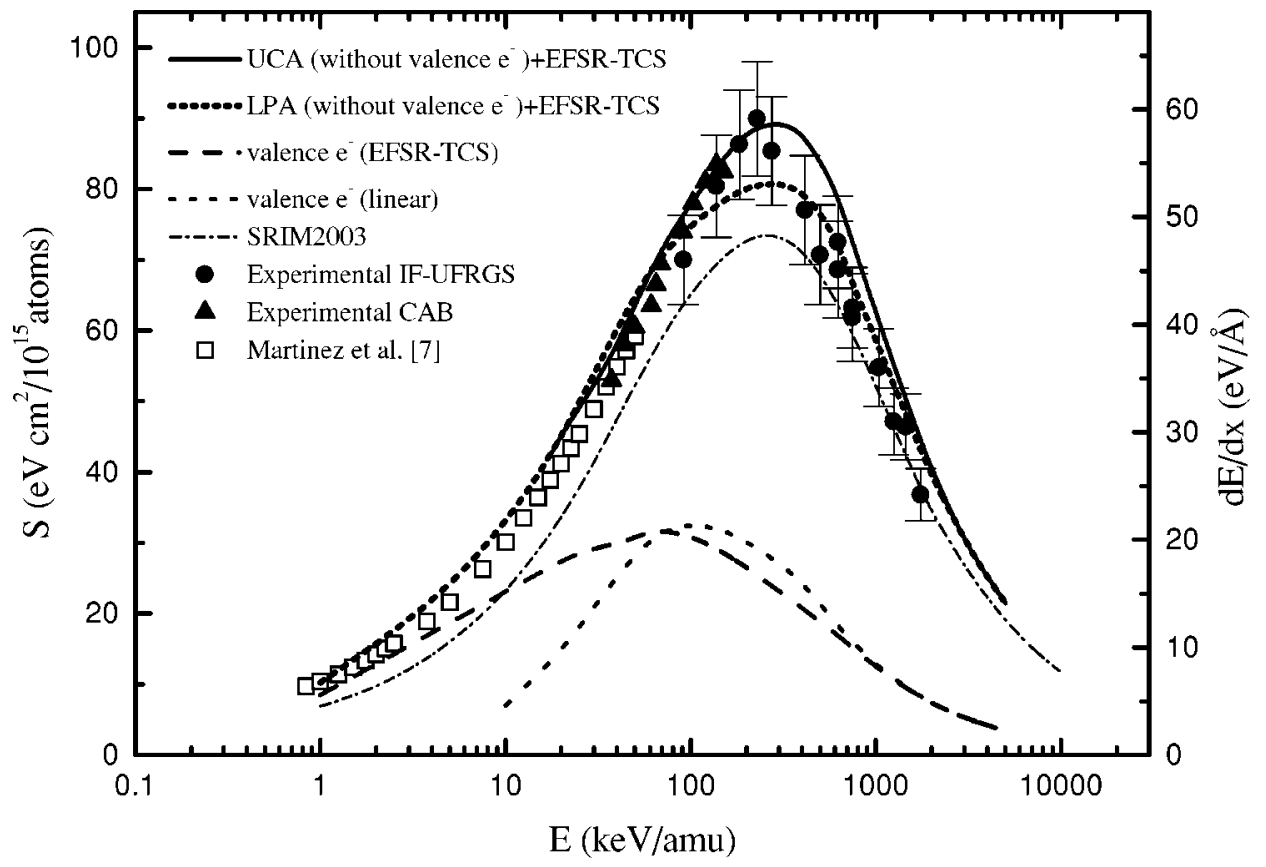

FIG. 3. Stopping powers of $\mathrm{He}$ in $\mathrm{Zn}$ as a function of energy. Symbols represent experimental measurements and lines represent theoretical calculations and the fitting curve of Ref. [5].

stopping in this region is due to the valence electrons (e.g., for energies below $5 \mathrm{keV}$ the contribution of the inner-shell electrons is less than $20 \%$, and stems mainly from the $M$ shell).

Also shown in Fig. 3 are the values from Ref. [5]. As it can be seen, the difference with the present values is substantial, reaching almost $20 \%$ in the region around the stopping power maximum.

\section{CONCLUDING REMARKS}

We present energy loss measurements of helium ions in $\mathrm{Zn}$, a metal of widespread technological interest covering an extended energy range and using different experimental techniques, as well as theoretical calculations using recent models. The results show the following features.

(1) A good agreement between the experiments made in both laboratories has been found, as well as a very good agreement with the calculations, showing that the inclusion of nonlinear effects in the theoretical models is required to achieve a satisfactory description of the measured energy loss.

(2) In the energy range covered in this work, the contribution of the inner-shell electrons increases from $\sim 50 \%$ at $37.5 \mathrm{keV} / \mathrm{amu}$ to $\sim 85 \%$ at $1.7 \mathrm{MeV} / \mathrm{amu}$.

(3) Energy loss values significantly higher than the SRIM 2003 predictions have been determined.

\section{ACKNOWLEDGMENTS}

This work was partially supported by the Argentine Agencia Nacional de Promoción Científica y Tecnológica, Projects PICT 03-03579 and PICT 03-06249, the Consejo Nacional de Investigaciones Científicas y Técnicas (PIP 4267/96), and the Cooperation Program SETCIP-CAPES 33/02 (BR/A U111/008) between Brazil and Argentina.
[1] H. Paul, D. Semrad, and A. Seilinger, Nucl. Instrum. Methods Phys. Res. B 61, 261 (1991).

[2] M. J. Berger, U.S. Department of Commerce, National Institute of Standards and Technology Report No. NISTIR 4999 1992 (unpublished).

[3] H. H. Andersen and J. F. Ziegler, Hydrogen Stopping Powers and Ranges in All Elements, edited by J. F. Ziegler, Stopping and Ranges of Ions in Matter, Vol. 3 (Pergamon, New York, 1977).

[4] J. F. Ziegler, Stopping Powers and Ranges in All Elements, edited by J. F. Ziegler, Stopping and Ranges of Ions in Matter, Vol. 4 (Pergamon, New York, 1977).

[5] J. F. Ziegler and J. P. Biersack, SRIM2003-The Stopping and
Range of Ions in Matter, Version 2003.23, code avaliable from www.srim.org.

[6] M. Luomajärvi, Radiat. Eff. 37, 223 (1978).

[7] G. Martinez-Tamayo, J. C. Eckardt, G. H. Lantschner, and N. R. Arista, Phys. Rev. A 54, 3131 (1996).

[8] A. Valenzuela and J. C. Eckardt, Rev. Sci. Instrum. 42, 127 (1971).

[9] J. C. Eckardt and G. H. Lantschner, Thin Solid Films 249, 11 (1994).

[10] P. Mertens, Nucl. Instrum. Methods Phys. Res. B 27, 315 (1987).

[11] W. K. Chu, J. W. Mayer, and M. A. Nicolet, Backscattering Spectrometry (Academic, New York, 1978).

[12] A. F. Lifschitz and N. R. Arista, Phys. Rev. A 57, 200 (1998). 
[13] A. L. Lifschitz and N. R. Arista, Phys. Rev. A 58, 2168 (1998).

[14] N. R. Arista, Nucl. Instrum. Methods Phys. Res. B 195, 91 (2002).

[15] A. F. Lifschitz and N. R. Arista, Phys. Rev. A 69, 012902 (2004).

[16] G. Schiwietz and P. L. Grande, Nucl. Instrum. Methods Phys. Res. B 175-177, 125 (2001).

[17] K. Shima, T. Mikuno, and H. Tawara, At. Data Nucl. Data Tables 34, 357 (1986); K. Shima, N. Kuno, M. Yamanouchi, and H. Tawara, At. Data Nucl. Data Tables 51, 173 (1992).

[18] D. Isaacson, New York University, Doc. No. 02698 (National Auxiliary Publication Service, New York, 1975).

[19] P. L. Grande and G. Schiwietz, Phys. Rev. A 58, 3796 (1998); G. Schiwietz and P. L. Grande, Nucl. Instrum. Methods Phys. Res. B 153, 1 (1999); G. M. de Azevedo, P. L. Grande, and G. Schiwietz, Nucl. Instrum. Methods Phys. Res. B 164, 203 (2000).
[20] P. L. Grande and G. Schiwietz, Convolution Approximation for Swift Particles, CasP version 1.2, code avaliable from www.hmi.de/people/schiwietz/casp.html.

[21] J. D. Fuhr, V. H. Ponce, F. J. García de Abajo, and P. M. Echenique, Phys. Rev. B 57, 9329 (1998).

[22] C. C. Montanari, J. E. Miraglia, and N. R. Arista, Phys. Rev. A 66, 042902 (2002).

[23] C. C. Montanari, J. E. Miraglia, and N. R. Arista, Phys. Rev. A 67, 062702 (2003).

[24] E. Clementti and C. Roetti, At. Data Nucl. Data Tables 14, 177 (1974).

[25] U. Kadhane, C. C. Montanari, and L. Tribedi, Phys. Rev. A 67, 032703 (2003).

[26] U. Kadhane, C. C. Montanari, and L. Tribedi, J. Phys. B 36, 3043 (2003).

[27] C. Reinhold and J. E. Miraglia, J. Phys. B 20, 1069 (1987).

[28] S. Heredia Avalos, Ph.D. thesis, Universidad de Murcia, 2002. 